Supporting Information for

\title{
Multi-Photon Fluorescence Quenching of Conjugated Polymers for TNT Detection
}

\author{
Aditya Narayanan ${ }^{\dagger}$, Oleg P. Varnavski, ${ }^{\ddagger}$ Timothy M. Swager, ${ }^{\ddagger}$ Theodore Goodson III ${ }^{* *}$ \\ ${ }^{\dagger}$ Department of Macromolecular Science and Engineering, ${ }^{*}$ Department of Chemistry, \\ University of Michigan, Ann Arbor, Michigan, 48109 and Institute for Soldier Nanotechnologies, \\ Massachusetts Institute of Technology, Cambridge MA 02139 \\ RECEIVED DATE (automatically inserted by publisher); *tgoodson@ umich.edu
}

\section{Optical Absorption and Emission Measurements}

The absorption spectra of the molecules were measured using an Agilent (Model \# 8341) spectrophotometer. The emission spectra were acquired using a Fluoromax-2 (SPEX) spectrophotometer. They are presented in Figure 1.

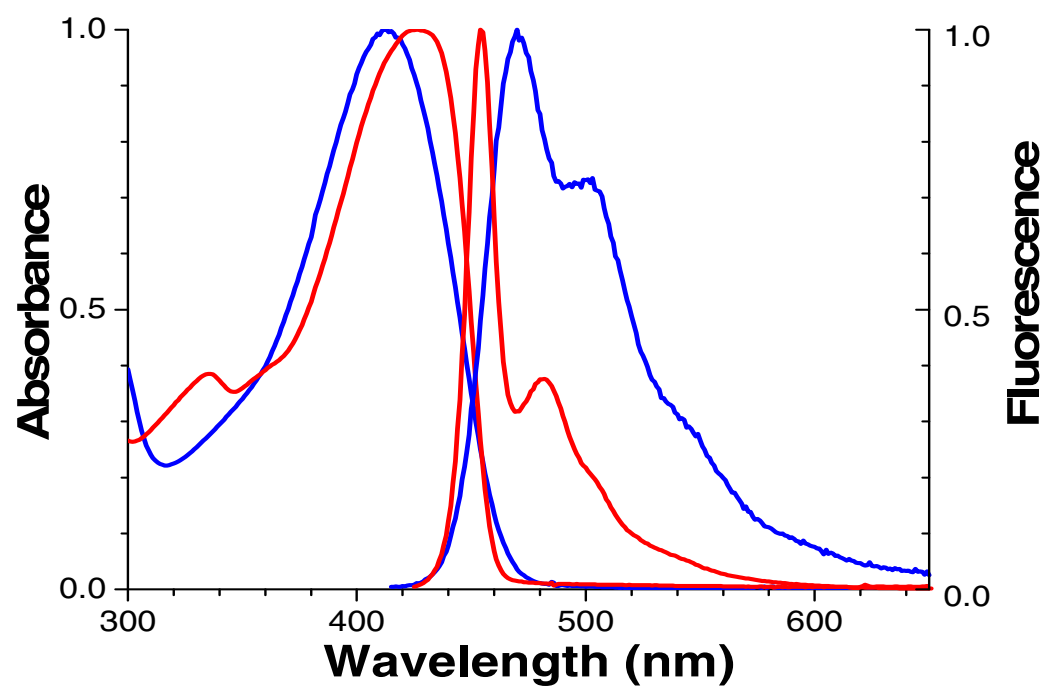

Figure 1. Normalized absorption and fluorescence spectra of $(A) P 1$, blue and (B) $P 2$, red, in Chloroform 


\section{Fluorescence Quantum Yield Measurements}

The quantum yields of the molecules were measured using a known procedure. ${ }^{1}$ Coumarin 307 was used as the standard. The solutions were purged with Argon for 3 minutes prior to measuring their emission spectra. Then, the following relation was used to measure the quantum yield:

$$
\phi_{F}=\left(\phi_{F}\right)_{S} \frac{\int J(\bar{v}) d \bar{v}}{\int J_{S}(\bar{v}) d \bar{v}} \frac{\left(J_{a}\right)_{S}}{J_{a}} \frac{n^{2}}{n^{2}{ }_{S}}
$$

where,

$\left(\phi_{F}\right)_{S} \quad=$ Quantum yield of the standard

$\int J(\bar{v}) d \bar{v}=$ Area under the fluorescence emission curve for the sample

$\int J_{S}(\bar{v}) d \bar{v}=$ Area under the fluorescence emission curve for the standard.

$\left(J_{a}\right)_{S} \quad=$ Absorbance of the standard

$J_{a} \quad=$ Absorbance of the sample

$n^{2} \quad=$ Refractive index of the solvent used for the sample.

$n_{s}^{2} \quad=$ Refractive index of the solvent used for the standard.

These measurements may have some error due to sensitivity of the fluorescence spectrophotometer and other environmental conditions.

\section{Multi-photon absorption cross-section measurements}

Based on the discussed experimental methods, the following relationship ${ }^{2}$ was used to calculate the two photon absorption cross-sections of the polymers.

$$
F(t) \approx \frac{1}{2} \eta \delta C \frac{8 g_{p}}{f \tau} \frac{\langle P(t)\rangle^{2}}{\pi \lambda} n \phi
$$

where,

$F(t)=$ Fluorescence intensity

$\eta \quad=\quad$ Fluorescence quantum yield

$\delta=\quad$ Two photon absorption cross-section

$C=$ Concentration of sample

$g_{p} \quad=\quad$ Laser repetition rate

$P(t)=$ Input Power intensity

$f \quad=\quad$ Frequency of Laser

$\tau=$ Pulse duration of Laser

$\lambda=$ Wavelength of excitation beam 
$n=$ refractive index of solvent

$\phi \quad=\quad$ Collection efficiency of system

The three photon absorption cross-section was also determined using a known relative method, ${ }^{3}$ which utilizes the following relation.

$$
\delta_{3 \mathrm{PA}}=\frac{3 \sqrt{3}}{4} \frac{\pi^{3}}{\ln 2} \frac{v_{3}{ }^{3}\left\langle I_{1}\right\rangle}{v_{1}\left\langle I_{3}\right\rangle^{3}} \frac{F_{3}}{F_{1}} \frac{C_{1}}{C_{3}} h^{2} r_{0}{ }^{4} \tau^{2} \sigma_{1}
$$

where,

$\delta_{3 P A}=$ Three photon absorption cross-section

$\sigma_{1} \quad=\quad$ One photon absorption cross-section measured at frequency $v_{1}$

$g=$ Laser repetition rate

$\tau=$ Pulse duration of Laser

$r_{0} \quad=\quad$ Diameter of pinhole

$h=$ Plank's constant

$C_{1}, C_{3}=$ Concentrations for one- and three-photon excitation respectively

$F 1, F 3=$ Intensities of one- and three-photon excited fluorescence respectively

$\left\langle I_{1}\right\rangle,\left\langle I_{3}\right\rangle=$ Average Laser intensities for one- and three-photon excitation respectively

\section{References in supporting information}

1. Maciejewski, A.; Steer;, R. P. J. Photochem. 1986, 35, 59.

2. Xu, C.; Webb, W. W.; J. Opt. Soc. Am. B. 1996, 13, 481.

3. Drobizhev, M.; Karotki, A.; Kruk, M.; Dzenis, Yu.; Rebane, A.; Suo, Z.;

Spangler, C.W. J.Phy.Chem. B , 2004, 108(14),4221. 